\title{
DOES THE DATA RESOLUTION/ORIGIN MATTER? SATELLITE, AIRBORNE AND UAV IMAGERY TO TACKLE PLANT INVASIONS
}

\author{
Jana Müllerováa ${ }^{\mathrm{a},}$, Josef Brůna ${ }^{\mathrm{a}, \mathrm{b}}$, Petr Dvořák ${ }^{\mathrm{c}}$, Tomáš Bartaloš ${ }^{\mathrm{d}}$, Michaela Vítkováa \\ ${ }^{a}$ Institute of Botany, The Czech Academy of Sciences, Průhonice, Czech Republic - (jana.mullerova, michaela.vitkova)@ibot.cas.cz \\ ${ }^{\mathrm{b}}$ Faculty of Science, Charles University in Prague, Czech Republic - josef.bruna@ibot.cas.cz \\ c Brno University of Technology, Institute of Aerospace Engineering, Brno, Czech Republic - dvorak.p@ fme.vutbr.cz \\ d GISAT Ltd., Prague, Czech Republic - tomas.bartalos@gisat.cz \\ * Corresponding author
}

ThS15

KEY WORDS: Detection; Invasive species; Mapping; MSS imagery; Object- and pixel-based classification; Spectral, spatial and temporal resolution

\begin{abstract}
:
Invasive plant species represent a serious threat to biodiversity and landscape as well as human health and socio-economy. To successfully fight plant invasions, new methods enabling fast and efficient monitoring, such as remote sensing, are needed. In an ongoing project, optical remote sensing (RS) data of different origin (satellite, aerial and UAV), spectral (panchromatic, multispectral and color), spatial (very high to medium) and temporal resolution, and various technical approaches (object-, pixelbased and combined) are tested to choose the best strategies for monitoring of four invasive plant species (giant hogweed, black locust, tree of heaven and exotic knotweeds). In our study, we address trade-offs between spectral, spatial and temporal resolutions required for balance between the precision of detection and economic feasibility. For the best results, it is necessary to choose best combination of spatial and spectral resolution and phenological stage of the plant in focus. For species forming distinct inflorescences such as giant hogweed iterative semi-automated object-oriented approach was successfully applied even for low spectral resolution data (if pixel size was sufficient) whereas for lower spatial resolution satellite imagery or less distinct species with complicated architecture such as knotweed, combination of pixel and object based approaches was used. High accuracies achieved for very high resolution data indicate the possible application of described methodology for monitoring invasions and their long-term dynamics elsewhere, making management measures comparably precise, fast and efficient. This knowledge serves as a basis for prediction, monitoring and prioritization of management targets.
\end{abstract}

\section{INTRODUCTION}

Invasive species have large effect on landscape, nature and human society (both economy and human health; Ehrenfeld, 2010), and despite the worldwide efforts, their menace grows (Hulme et al., 2009). Invasions represent fast and dynamic processes and their eradication and control is difficult. Early and regular detection of the invading species and rapid management response on both local and landscape level is therefore crucial (Vilà and Ibáñez, 2011). There is an urgent need for new techniques enabling timely, fast and precise monitoring (European Commission, 2008). Remote sensing (RS) can potentially provide detailed vegetation mapping over large areas, reducing costs of extensive field campaigns. Multispectral optical data have been successfully used to study invasive plant species (for review see Huang and Asner, 2009), however, mostly for shrubs and trees (but see e.g. Müllerová et al., 2013; Jones et al., 2011). Detection of herb species is only possible if the data provide enough spectral and/or spatial details, the species are distinct from their neighborhood, form dense and uniform stands, and/or are large enough to be detected (Maheu-Giroux and de Blois, 2004; Müllerová et al., 2005). The potential for invasive species monitoring using RS is still not fully exploited and for the majority of invasive species there are no detection algorithms described.

In an on-going project, we aim to meet the need for costeffective techniques for timely, fast and precise management including prevention and monitoring measures of alien plant species. We are searching for the solution that would be operational and applicable for landscape managers regarding both costs and labor. Testing various RS data gives us opportunity to evaluate potential of data differing in spectral, spatial and temporal resolution, and to choose best mapping strategies for species of interest. Using UAV provides flexibility necessary for assessment of role of phenology crucial for detection of species distinct only in certain phenological stages, e.g. during flowering. Such evaluation could not be done using any other source of RS data. To make the use of UAV for invasion detection as operational as possible, we seek for lowcost solution, developing purposely designed UAV for the data acquisition. Methodology developed for automatic/semiautomatic detection of four selected invasive species will help to minimize the negative impact of invasions and save management costs by providing means for fast and timely monitoring of plant invasions.

\section{METHODS}

\subsection{Study species}

Species of interest are: giant hogweed (Heracleum mantegazzianum), black locust (Robinia pseudoaccacia), tree of heaven (Ailanthus altissima), and exotic knotweeds (Fallopia japonica, $F$. sachalinensis and $F . \times$ bohemica). All mentioned species are considered invasive in a number of European 
countries and North America (except for the American native black locust), and are listed among the hundred most aggressive invaders in Europe (DAISIE database, http://www.europealiens.org/).

Tree of heaven is a fast growing deciduous tree $20 \mathrm{~m}$ tall, native to East Asia and introduced worldwide. It is a species of warmer regions with potential to spread due to global warming. It can be found in urban environments, industrial zones, along railways, but also in termophilous shrub and grassland vegetation. It causes loss of biodiversity, its root system damages pavements, walls and buildings and it poses danger for humans, because its sap is causing contact dermatitis.

Exotic knotweeds are herbaceous perennials with robust erect stems up to $4 \mathrm{~m}$ tall, native to East Asia, and their hybrid $F . \times$ bohemica. They form dense monospecific stands on nutrient rich mesic to wet sites in settlements, abandoned gardens and floodplains. They cause a loss of biodiversity, alteration of hydrological regime, damage of roads and floodprevention structures and increase erosion potential of rivers.

Giant hogweed is a herbaceous perennial plant up to $4 \mathrm{~m}$ tall with large round inflorescences up to $2 \mathrm{~m}$ in diameter, native to the Western Greater Caucasus. It often forms large monospecific stands in semi-natural grasslands, forest edges and anthropogenic habitats along roads. As a mountain plant, it rarely occurs in dry and warm lowlands. It causes a loss of biodiversity and is dangerous to humans, because its sap is causing dermatitis.

Black locust is a deciduous tree up to $30 \mathrm{~m}$ tall native to southeast of the USA. It was planted for timber, erosion control, soil eutrophication and honey making. As nitrogen fixing tree it changes availability of soil nutrients and causes vegetation changes towards ruderal and nitrophilous species resulting in loss of biodiversity. It is toxic to cattle and horses.

Whereas the RS detection of the tree of heaven was not previously investigated, for other three species the timing of the data acquisition showed to be important (exotic knotweeds: Dorigo et al., 2012; Jones et al., 2011; g. hogweed: Müllerová et al., 2005, 2013; b. locust: Somodi et al., 2012).

\subsection{Remote sensing data}

Optical RS data of different origin (satellite, aerial and UAV), spectral (panchromatic, multispectral and color), spatial (very high to medium) and temporal resolution were tested in our project.

\subsubsection{Satellite and aerial data}

Optical RS data of different resolution were chosen to assess the importance of spectral/spatial resolution for the species recognition. Following data were used in the study (Figure 1):

- $\quad$ panchromatic historical aerial photography $(0.5 \mathrm{~m})$

- color aerial orthophotos $(0.5 \mathrm{~m})$

- World View-2 (0.5/1.84 m PAN/MSS)

- World View-3 (0.3/1.24 m PAN/MSS)

- Pleiades $(0.5 / 2.8 \mathrm{~m}$ PAN/MSS resolution)

- $\quad$ RapidEye $(6.5 \mathrm{~m})$

- Sentinel-2 (10 m)
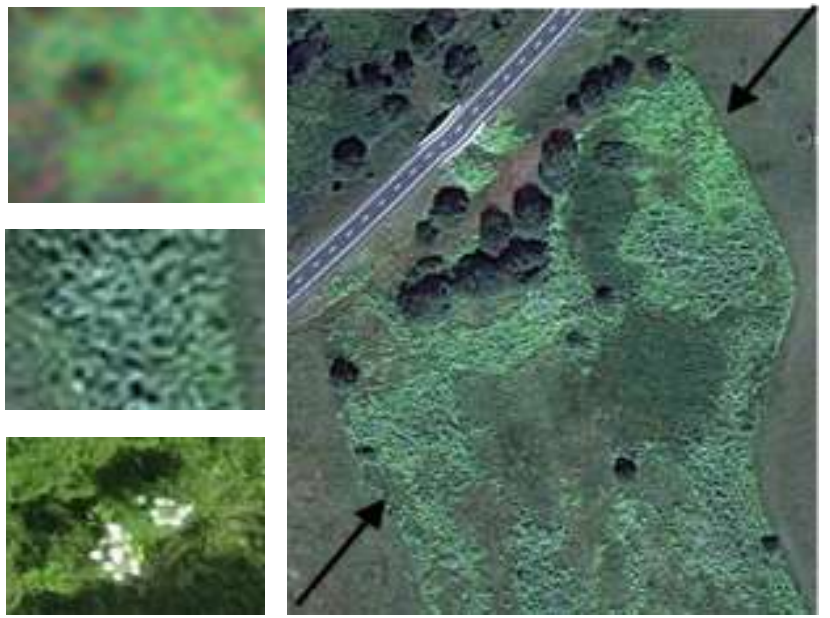

Figure 1. Giant hogweed on Pleiades satellite imagery (right). On the left are details of flowering plants captured by RapidEye, Pleiades and UAV (from up to down, spatial resolution of 6.5 $\mathrm{m}, 2.8 \mathrm{~m}$ and $5 \mathrm{~cm}$ ).

\subsubsection{Unmanned aircraft}

Acquisition of UAV data has been performed by several aerial platforms (Table 1). Development of dedicated unmanned platform optimized for invasive species monitoring is an ongoing task of the project (Dvoř́k et al., 2015).

\begin{tabular}{|c|c|c|c|}
\hline & VUT 712 & VUT 713 & VUT 720 \\
\hline Span & $2.1 \mathrm{~m}$ & $2.0 \mathrm{~m}$ & $2.6 \mathrm{~m}$ \\
\hline Length & $0.9 \mathrm{~m}$ & $0.7 \mathrm{~m}$ & $1.3 \mathrm{~m}$ \\
\hline mтоW & $3.1 \mathrm{~kg}$ & $3 \mathrm{~kg}$ & $2.2 \mathrm{~kg}$ \\
\hline $\mathrm{v}_{\mathrm{C}}$ & $17 \mathrm{~m} / \mathrm{s}$ & $18 \mathrm{~m} / \mathrm{s}$ & $15 \mathrm{~m} / \mathrm{s}$ \\
\hline Endurance & $0.9 \mathrm{hr}$ & $0.8 \mathrm{hr}$ & $1 \mathrm{hr}$ \\
\hline Power & $800 \mathrm{~W}$ & $600 \mathrm{~W}$ & $360 \mathrm{~W}$ \\
\hline Payload & $0.8 \mathrm{~kg}$ & $0.9 \mathrm{~kg}$ & $0.3 \mathrm{~kg}$ \\
\hline Autopilot & $\begin{array}{l}\text { Pixhawk } \\
\text { autopilot }\end{array}$ & $\begin{array}{l}\text { Pixhawk } \\
\text { autopilot }\end{array}$ & $\begin{array}{l}\text { APM2.5+ } \\
\text { autopilot }\end{array}$ \\
\hline Camera & $\begin{array}{l}\text { 2x Canon } \\
\text { S100 } \\
\text { stabilized }\end{array}$ & $\begin{array}{c}2 \mathrm{x} \text { Sony } \\
\text { A5100 + } \\
\text { E20/2.8 }\end{array}$ & $\begin{array}{c}\text { 1x Canon } \\
\text { S100+ } \\
\text { 1x GoPro }\end{array}$ \\
\hline Based on & $\begin{array}{c}\text { SkyWalker } \\
\text { X8 }\end{array}$ & RVJET & $\begin{array}{c}\text { Multiplex } \\
\text { Cularis }\end{array}$ \\
\hline
\end{tabular}

Table 1. Small VUT unmanned platforms for invasive species monitoring used in the project

For the initial trials a motorised glider concept was selected (VUT720, Dvorak et al., 2013, Figure 2) to take advantage of the low wing loading. Combined with expanded polypropylene, a durable material featuring excellent impact properties, this concept ensures safe landings even in rugged terrain. The platform is hand-launched with no need for additional equipment. 


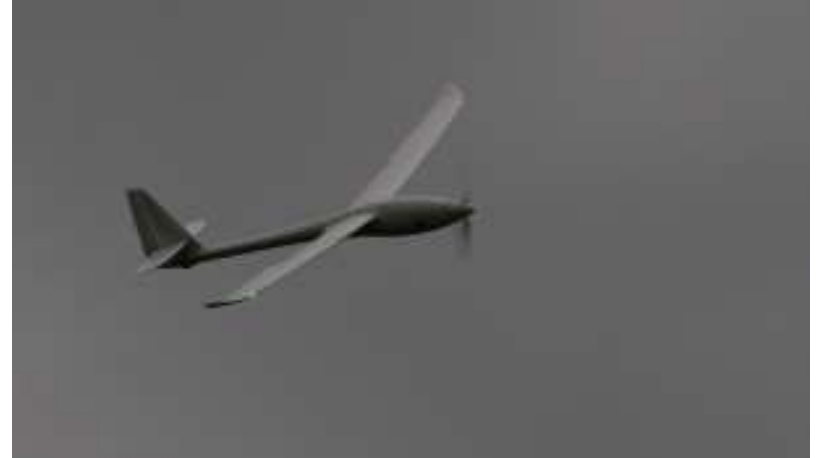

Figure 2. VUT 720 in flight

However, VUT720 is able to carry only one camera (Canon PowerShot S100) at a time. The requirement to produce RGB+NIR data for further classification and research meant that two consecutive flights had to be performed at each location. This procedure not only resulted in increased time spent at a single site, but also introduced significant problems during image processing, as the lighting conditions can often change during consecutive flights making the radiometric corrections of the captured images more complex. Therefore a platform providing actively stabilized mount for two Canon S100 cameras is concurrently employed - VUT712 (Figure 3). Data acquired with VUT712 prove superior to previous datasets, both in terms of radiometric consistency as well as georeferencing accuracy.

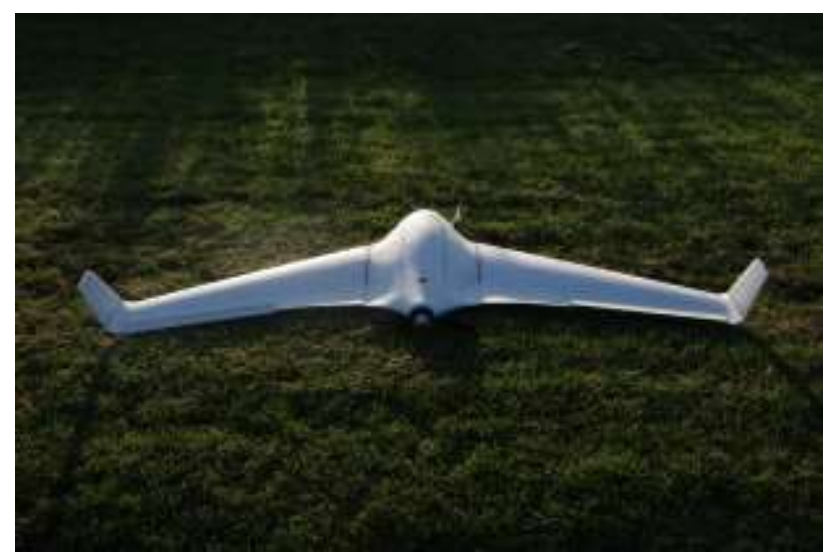

Figure 3. VUT 712

Operational experience clearly shows that consumer point-andshoot camera such as Canon S100 is not well suited for the harsh environmental conditions of repeated mapping missions.

Retractable zoom lens of the camera are inherently prone to (uncommanded) focal length changes, deteriorating the photogrammeteric quality of acquired pictures. Dust can penetrate the lens easily during landings, resulting in jammed lens and prolonged down-times of the platform. The sensor size of 1/1.7" (7.44 x $5.58 \mathrm{~mm})$ results in comparably high levels of noise and low dynamic range of the pixels. NIR modified camera is especially affected by this fact due to the longer wavelength of sensed light.

Deployment of dedicated multispectral cameras (such as Micasense RedEdge) or an array of industrial grade cameras with spectral filters would overcome mentioned problems, however the cost would limit operational use. As a costeffective solution, fixed pancake lens (Sony E20/2.8) coupled to a lightweight mirrorless camera (Sony A5100) has been deployed in a new platform - VUT 713. The APS-C (23.5 x $15.6 \mathrm{~mm}$ ) sized sensor of this camera provides nearly 8.8 times greater photosensitive area compared to the S100 camera. Fixed lens addresses the problems experienced with retractable zoom lens. This setup has yet to be proven by long-term operational deployment.

\subsection{Geometric processing of the UAV data}

During the UAV flights campaigns several sets of imagery were acquired within varied conditions (e.g. illumination or altitude dependant) to test and adapt the optimal configuration. The subsequent workflow for image processing includes SfM (structure-from-motion) algorithm implemented within Agisoft Photoscan software. The main advantage of this method is automatic and simultaneous solution of scene geometry and camera position and orientation (Westboy et al., 2012). The software also allows scripting for batch processing. All acquired spectral channels are processed in one step, resulting in fully co-registered VIS+NIR mosaic (Figure 4) and detailed digital surface model (DSM, Figure 5).

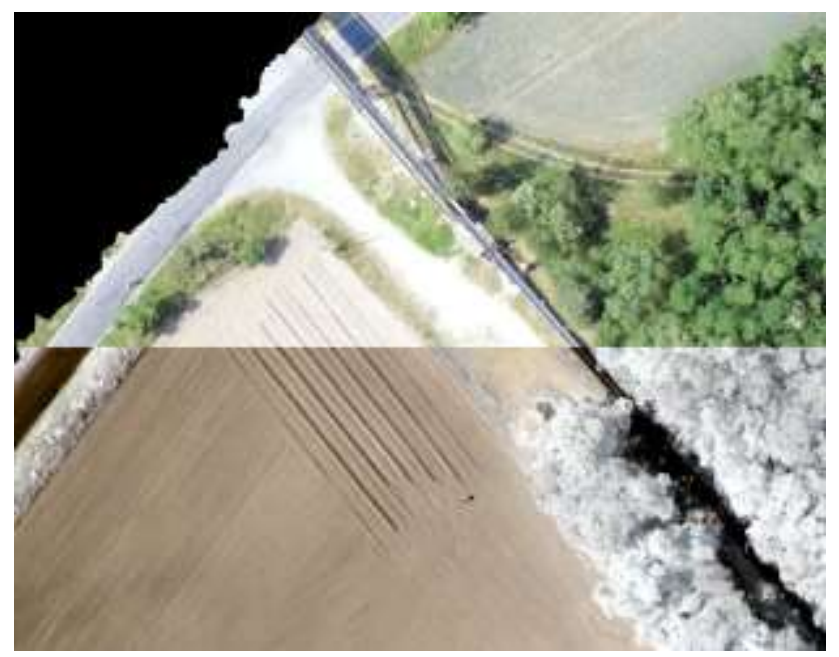

Figure 4. Precise geometric co-registration of VIS and NIR spectral channels

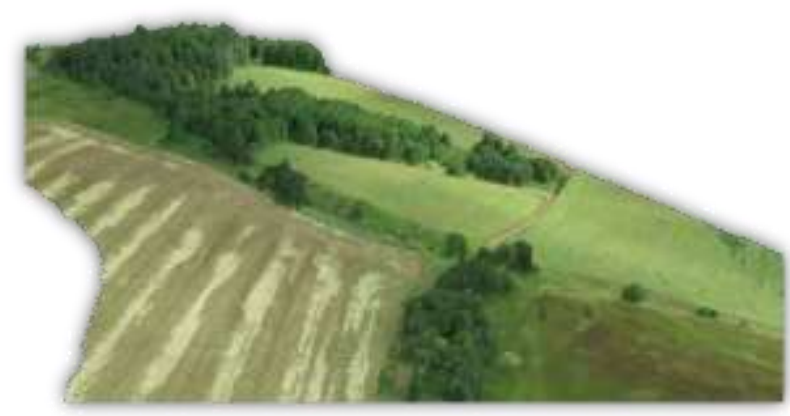

Figure 5. Digital surface model generated from UAV imagery by SfM algorithm

For improvement of the final orthophoto mosaic further testing of input imagery is considered. Filtration of images based on flight data analysis improved the selection of potentially distorted images such as blurred images or images obtained at high angle. Images evaluated as non-satisfactory are to be excluded from additional processing. However, the preliminary results show very low number of such images. Oblique images occur usually at edges of the area of interest, and do not 
influence image mosaic quality inside the true target area significantly.

The test flights and current generated ortho-mosaics suffer from significant geometrical inaccuracies (up to $\mathrm{x} 10 \mathrm{~m}$ ) in comparison to reference data (aerial orthophoto). These are mainly caused by using low-cost consumer camera GPS information only. To improve the positional accuracy, needed for precise delineation of target species, improvement of GPS information based on data extracted from autopilot telemetry data was tested. However the preliminary tests proved not to be satisfactory and precise enough besides the problematic synchronization and proper time alignment (Figure 6).

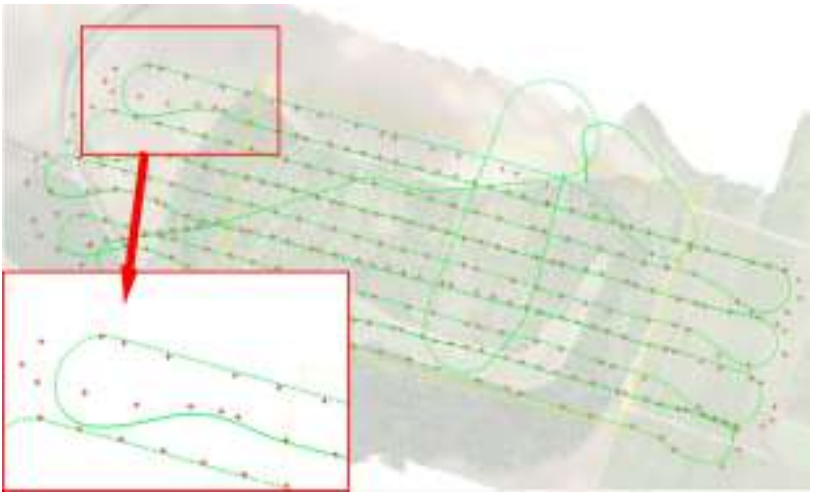

Figure 6. Comparison of GPS coordinates of camera (red cross) and autopilot (green line)

Alternatively, GCP points were collected within selected sites with measured exact GPS coordinates using differential GPS to be used during automatic orthorectification of scenes. This arrangement significantly improved the final geometric accuracy. To further enhance the accuracy, more accurate GPS receiver/GNSS-INERTIAL unit will be obtained for the platform. For optimal processing, extended parameter test was performed to evaluate optimal settings and configuration with regard to final mosaic quality (elimination of artefacts) and processing time (Figure 7).
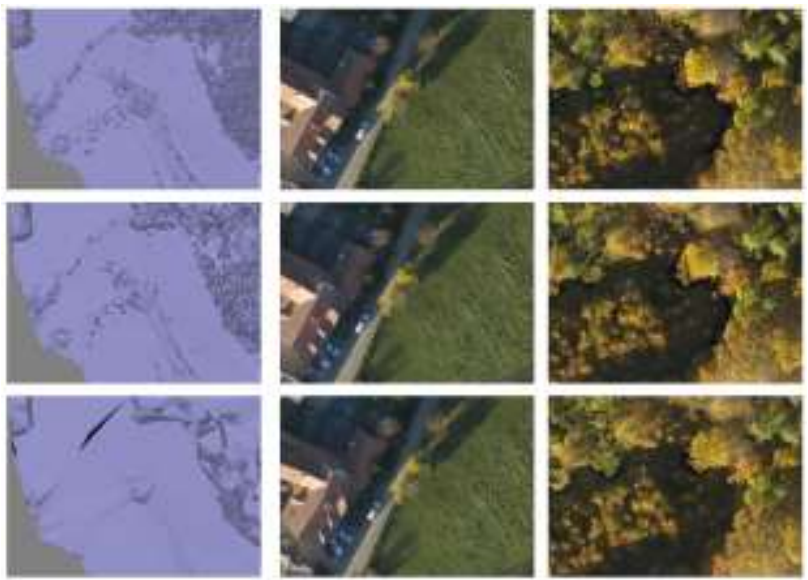

Figure 7. Example of mosaicking result with different set of mesh parameters for surface model

\subsection{Image analysis}

Both pixel and object based approaches are applied using eCognition Developer, PCI Geomatica and ArcGIS software.
For pixel-based approach, both unsupervised (ISODATA, Kmeans and Fuzzy K-means) and supervised (maximum likelihood and minimum distance (Jensen, 2004) classifications are tested. For object-based approach, multiresolution segmentation is applied with parameters (scale, shape and compactness) determined using a systematic trial-and-error approach. Various scales are tested and segmentation outputs visually evaluated to identify the best parameters to extract the targets. Ruled-based classification (Blaschke et al., 2008) is applied on the segmented objects using conditions related to spectrum (brightness, mean layer, maximum pixel value, standard deviation, hue, saturation, intensity transformation for RGB), shape (area, length/width), texture (compactness, GLCM homogeneity, GLCM dissimilarity, and GLCM contrast), and context (contrast and edge contrast of neighbour pixels). For problematic, less distinct species such as knotweeds, hybrid approach considering the inter-object variability is tested. Segmentations objects of known, ground truth identified invasive species of interest were compared with objects with other vegetation cover to find significant differences among all object and pixel based variables. For knotweed classification on autumn UAV imagery, high reflectance in NIR was identified as the most reliable parameter together with high NDVI and overall brightness and maximum difference in an object. In the resulting potential objects, lower GLCM Entropy of knotweed objects was used to contrast them against remaining vegetation.

Ground-truth data were used for development of classification algorithms and evaluation of classification accuracy. Very high spatial resolution of UAV data generated problems with position accuracy of ordinary GPS used during field survey for ground truth data collection. Differential GPS would help to solve this problem, yet is very time consuming and costly. To make the methodology operational, we used consumer tablets with Collector for ArcGIS application installed to mark position of each invasive plant individual or patch in the field directly into the imagery (either aerial or UAV). Main reason for choosing this application was capability to: (i) collect the data offline and synchronize the results later and (ii) use offline image layers as base maps. This allowed us to both digitize the exact location of invasive species on ortophotos with higher spatial accuracy compared to GPS measurements, and map visible but inaccessible areas. We have also recorded and a details photograph of each site.

\section{RESULTS AND DISCUSSION}

\subsection{Detection success}

In our project, we use data combining different spatial, spectral and temporal resolution. These are: (i) very low spectral/very high spatial resolution data (panchromatic aerial photographs), (ii) medium spectral/very high spatial resolution (color aerial photographs), (iii) medium spectral/ultra-high spatial resolution (UAV RGB+NIR data) providing also high inter-annual temporal resolution, (iv) high spectral/medium spatial resolution (RapidEye, Sentinel satellite imagery), and (v) high spectral/very high spatial resolution (Pleiades, WorldView satellite imagery).

Our results are only preliminary; however we can infer that certain rules can be established for choosing the right data and approaches depending on the species to be detected. In case the target species is not spectrally distinct but the data spatial resolution is higher than the size of mapped individuals, the species can be recognized if forming unique spatial/spectral patterns. The object-based approach relying on both spectral and spatial information provides the best solution for low 
spectral resolution imagery such as historical panchromatic photography (see Laliberte et al., 2004, or Müllerová et al., 2013), less spectrally distinct species, and species with typical distinct shape pattern (Figure 8).
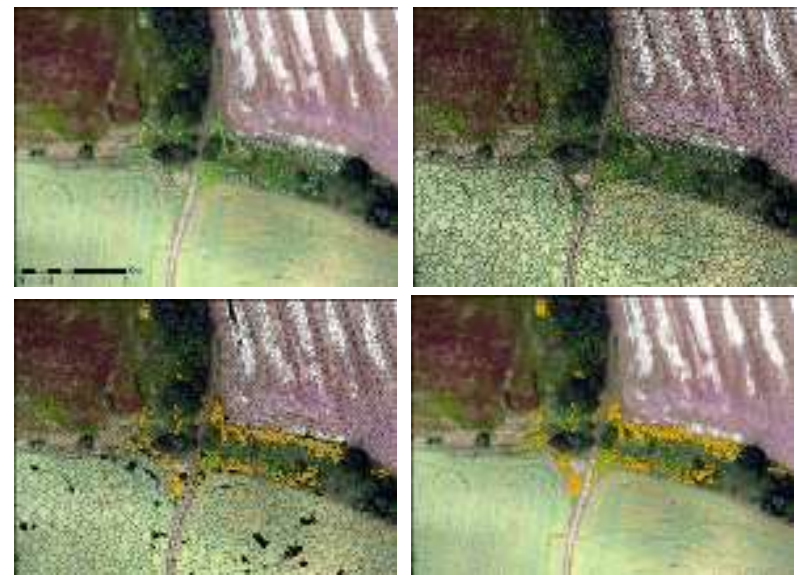

Figure 8. Detection of giant hogweed from UAV imagery using object oriented approach (resulting g. hogweed class is marked in orange)

In case the objects are smaller than the pixel size, objectoriented approach would not bring much advantage over pixelbased one. In this case, species can be successfully detected if forming homogeneous stands (and ideally holding unique spectral characteristics). Most species are distinct only at certain phenological stage (typically flowering), and temporal data can be best acquired by UAVs (Figure 9). Such source typically provides imagery of lower spectral resolution; however additional temporal layers can increase the detection accuracy. If only one survey can be obtained, right timing based on phenology of the species is critical.
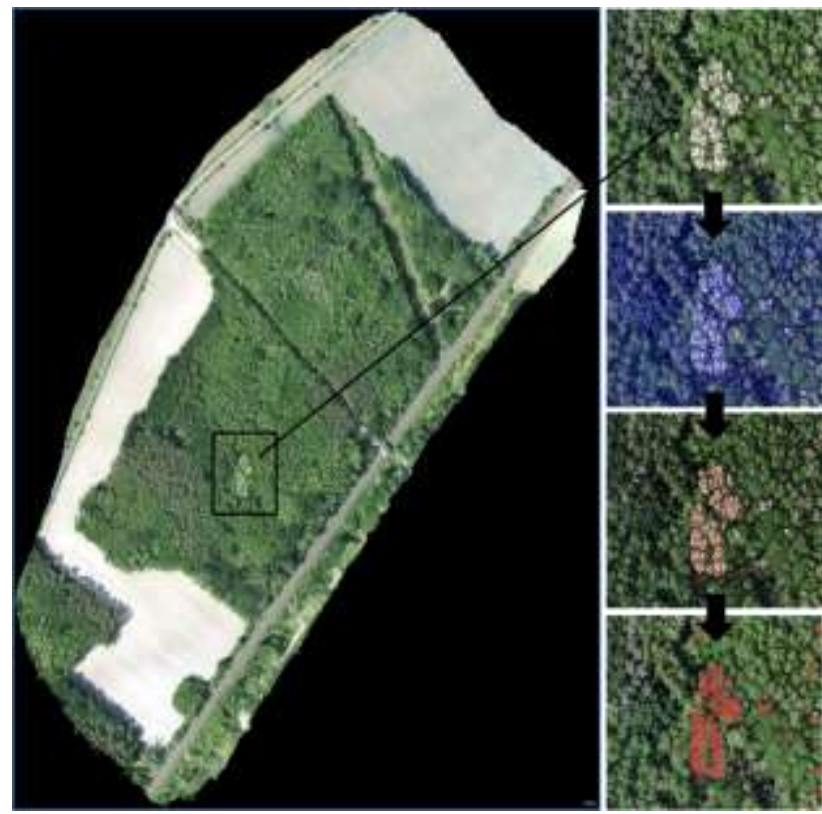

Figure 9. Process of black locust detection from UAV imagery using object-oriented approach, taking advantage of flowering phase, when the tree is well distinguished from surrounding tree species (resulting locust class is marked in red)

On very high spatial resolution UAV imagery, even small patches and individual plants can be recognized, helping the monitoring of the invasion onset and the dispersal foci, but due to the small areal extend of UAV images, large amount of data is needed to be processed which is labour and time intensive. Thankfully many of the tasks can be automated, which is also a goal of our project. On the other hand, satellites provide standardized datasets with large area covered by a single image, making the processing comparably faster, yet the spatial and temporal resolution might not be sufficient for some species (Figure 10). Satellite data availability can be limited due to the cloud cover, whereas flexible UAV can overcome such problem.

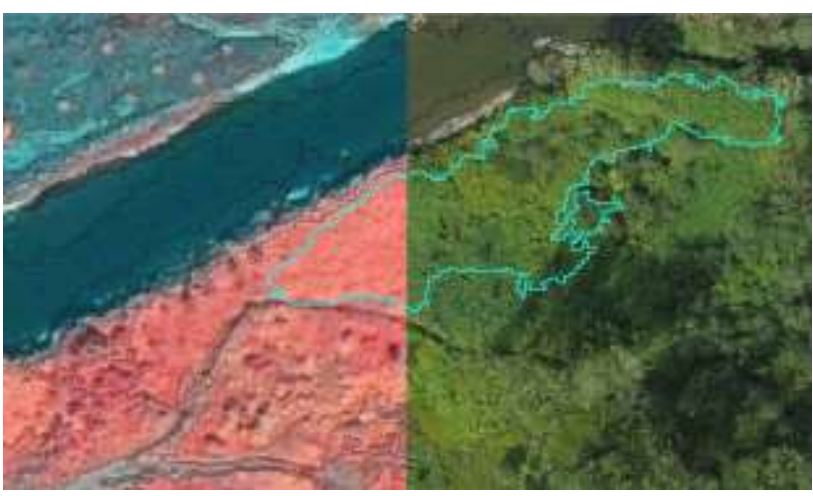

Figure 10. Pleiades 1B (false color composite of NIR-G-B, July 2015) vs. UAV (RGB, August 2015) imagery with segmented objects, blue outlines a knotweed patch identified from Pleiades Imagery.

\subsection{Implications for practical management, lessons learned}

When considering using RS for invasive species detection, it is necessary to be aware of its limits, e.g. no detection under the canopy, if mown (grazed), or if species is less distinct.

The successful mapping strategy must reflect plant morphological, structural and often most importantly phenological features. Choice of the data depends on both purpose and target - medium spatial resolution can give fast overview, whereas VHR (UAV) data provide details on invasion progress and early detection. For the less distinct species, higher resolution data are needed compared to the distinct one, with lower spectral possibly compensated by higher spatial resolution.

Best method must consider species characteristics - spectrally distinct ones can be successfully detected using pixel-based approach (such as g. hogweed during flowering), whereas species with distinct shape should be classified using objectbased approach where shape, texture and context is useful even when spectral resolution is low (e. g. hogweed on panchromatic imagery). For less distinct species such as knotweed or tree of heaven, combined approach might help to augment the resulting accuracies.

Acceptable accuracy levels depend on the purpose - maximized accuracy is needed to locate infestation hotspots, whereas lower accuracy is acceptable to cover all infested localities. For land managers, the results should minimize the error of omission, because for invasion mitigation, targeting every possible occurrence is vital for success of eradication campaigns. Remote sensing is used here as a way to minimize the area required for field inspection, which can be simply done during eradication on selected sites.

Purpose build UAV is a low cost solution providing very high spatial detail, high flexibility enabling optimal timing of the campaign (choosing the best phenological stage to detect the 
species). It can be successfully used for early detection and targeted monitoring (e.g. to survey sites prone to invasion, areas of interest for nature conservation, such as NATURA 2000 sites), or to check eradication success). On the other hand, it produces large amounts of data generating needs for automatization. Also the UAV data processing is more complex and demanding compared to standard satellite imagery. Using UAV for monitoring also has legal constrains that need to be considered. Within EU, UAV operation in urban and inhabited areas is prohibited, limiting the application especially for invasive species that thrive on man-made habitats. Despites its limits, it still provides a reasonable alternative to satellite imagery especially in vegetation mapping, where often data of high spatial and temporal resolution are required.

\section{CONCLUSIONS}

RS approach, namely application of UAV, represents fast, efficient, rather objective and repeatable method for detection, monitoring and studying plant invasions, reducing considerably the costs if compared to the field campaigns. However, appropriate species specific methodological approaches must be established to make the monitoring useful.

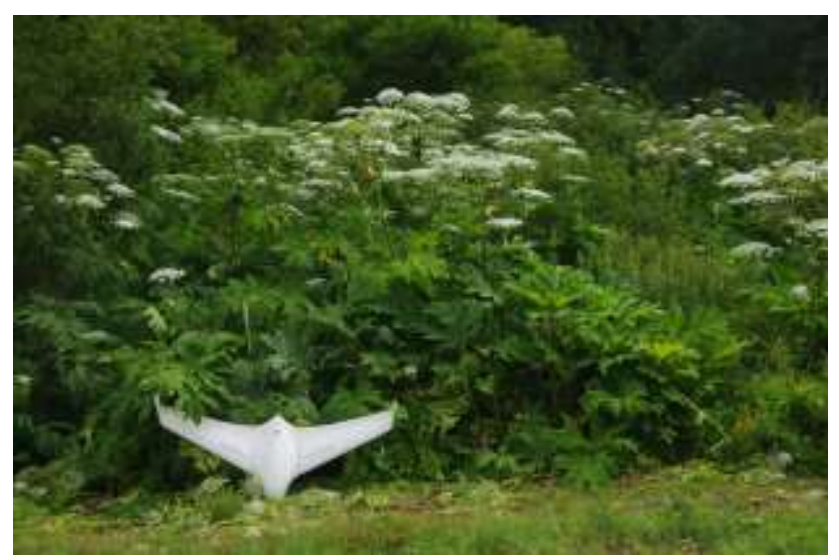

\section{ACKNOWLEDGEMENTS}

The research leading to these results has received funding from the MEYS under the National Sustainability Programme I (Project LO1202), the long-term research development project no. RVO 67985939 and project no. TA04020455.

\section{REFERENCES}

Blaschke, T., Lang, S., Hay, G.J. (Eds.), 2008. Object-based Image Analysis: Spatial Concepts for Knowledge-driven Remote Sensing Applications. Springer, Berlin.

Dorigo, W., Lucieer, A., Podobnikar T. \& Čarni A., 2012. Mapping invasive Fallopia japonica by combined spectral, spatial, and temporal analysis of digital orthophotos. International Journal of Applied Earth Observation and Geoinformation 19, pp. 185-195.

Dvorak, P., Pejchar, J. \& Zikmund, P., 2013. Overview of Unmanned Aerial Systems Developed at the Institute of Aerospace Engineering. In J. Juracka, A. Kazda, A. Novak (Eds.): New Trends in Civil Aviation 2013. Conference Proceedings. Brno, CERM, pp. 16-21.
Dvořák, P., Müllerová, J., Bartaloš, T., \& Brůna, J., 2015. Unmanned aerial vehicles for alien plant species detection and monitoring. Int. Arch. Photogramm. Remote Sens. Spatial Inf. Sci., XL-1/W4, pp. 83-90.

Ehrenfeld, J.G., 2010. Ecosystem consequences of biological invasions. Annual Review of Ecology, Evolution and Systematics 41, pp. 59-80.

European Commission, 2008. Towards an EU strategy on invasive species. COM, 789, EC, Brussels.

Huang Ch. \& Asner G.P., 2009. Applications of remote sensing to alien invasive plant studies. Sensors 9, pp. 4869-4889.

Hulme, P.E., Pyšek, P., Nentwig, W. \& Vilà, M., 2009. Will threat of biological invasions unite the European Union? Science 324. pp. 40-41.

Jensen, J.R., 2004. Introductory Digital Image Processing: A Remote Sensing Perspective, 3rd ed. Prentice Hall, London.

Jones D., Pike S., Thomas M. \& Murphy D., 2011. Objectbased image analysis for detection of Japanese Knotweed s.l. taxa (Polygonaceae) in Wales (UK). Remote Sensing 3, pp. 319-342.

Laliberte, A.S., Rango, A., Havstad, K.M., Paris, J.F., Beck, R.F., McNeely, R., Mathieu, R., Freeman, C., Aryal, J., 2004. Object-oriented image analysis for mapping shrub encroachment from 1937 to 2003 in southern New Mexico. Remote Sensing of Environment 93, pp. 198-210.

Maheu-Giroux M. \& de Blois S., 2004. Mapping the invasive species Phragmites australis in linear wetland corridors. Aquatic Botany 83, pp. 310-320.

Müllerová J., Pyšek P., Jarošík V. \& Pergl J., 2005. Aerial photographs as a tool for assessing the regional dynamics of the invasive plant species Heracleum mantegazzianum. Journal of Applied Ecology 42, pp. 1-12.

Müllerová J., Pergl J. \& Pyšek P., 2013, Remote sensing as a tool for monitoring plant invasions: testing the effects of data resolution and image classification approach on the detection of a model plant species Heracleum mantegazzianum (giant hogweed). International Journal of Applied Earth Observation and Geoinformation 25, pp. 55-65.

Somodi I., Čarni A., Ribeiro D. \& Podobnikar T., 2012. Recognition of the invasive species Robinia pseudacacia from combined remote sensing and GIS sources. Conserv. Biol. 150, pp. 59-67.

Vilà M. \& Ibáñez I., 2011. Plant invasions in the landscape. Landscape Ecology 26, pp. 461-472.

Westboy, M. J., Brasington, J., Glasser, N.F., Hambrey, M. J. \& Reynolds, J. M., 2012. 'Structure-from-Motion' photogrammetry. A low-cost, effective tool for geoscience applications. Geomorphology 179, pp. 300-314. 